\title{
Geometrical issues in the continuum mechanics of defective solid crystals
}

\author{
Rachel Nicks and Gareth Parry
}




\title{
GEOMETRICAL ISSUES IN THE CONTINUUM MECHANICS OF DEFECTIVE SOLID CRYSTALS
}

\author{
RACHEL NICKS AND GARETH PARRY
}

\begin{abstract}
We shall outline geometrical and algebraic ideas which appear to lie at the foundation of the theory of defective crystals that was introduced by Davini [5] in 1986. The focus of the paper will be on the connection between continuous and discrete models of such crystals, approached by consideration of the symmetries inherent in these models. To begin with, we review briefly the results of analysis of variational problems where relevant functionals have the symmetry of perfect (as opposed to defective) crystals, in order to motivate the subsequent study of symmetry in the case when defects are present. In the body of the paper we indicate how the theory of Lie groups, and their discrete subgroups, relates to this geometrical theory of defects, and discuss types of symmetry that occur.
\end{abstract}

2000 Mathematics Subject Classification: 74A20; 74E25

Keywords: crystal, Lie groups, discrete subgroups, symmetry

\section{INTRODUCTION}

In Davini's [5] model of defective crystals, the kinematical state of the crystal corresponds to a distribution of three vector fields in a region $\Omega \subseteq \mathbb{R}^{3}$. Thus the defective crystal state, $\Sigma$ say, is represented by

$$
\Sigma=\left\{\ell_{a}(\cdot), \Omega ; \quad a=1,2,3\right\},
$$

where $\ell_{1}(\cdot), \ell_{2}(\cdot), \ell_{3}(\cdot)$ are three 'lattice' vector fields defined and linearly independent at each $x \in \Omega$. One imagines that these three smooth fields also determine the local discrete structure at any point $x \in \Omega$. Thus, in the particular case where the crystal is 'perfect', the lattice vector fields are independent of the point $x, \ell_{a}(x)=e_{a}$ say, $\boldsymbol{x} \in \Omega, a=1,2,3$, where $\boldsymbol{e}_{1}, \boldsymbol{e}_{2}, \boldsymbol{e}_{3}$ is a basis of $\mathbb{R}^{3}$, and it is common to associate the perfect crystal lattice

$$
L \equiv\left\{\boldsymbol{x}: \boldsymbol{x}=n_{1} \boldsymbol{e}_{1}+n_{2} \boldsymbol{e}_{2}+n_{3} \boldsymbol{e}_{3}, n_{1}, n_{2}, n_{3} \in \mathbb{Z}\right\},
$$

(the translational symmetries of which are $\boldsymbol{e}_{1}, \boldsymbol{e}_{2}, \boldsymbol{e}_{3}$ ) with these 'constant' fields. The manner in which states $\Sigma$ determine corresponding discrete structures, in the general case, will be made explicit later.

\footnotetext{
The second author is the corresponding author.
} 
So far as continuum mechanical theories based on kinematical models such as (1.1) are concerned, it is a central task to prescribe how quantities such as stress, energy, etc., depend on the geometrical fields - this is a specification of the constitutive behaviour of the material. We shall not be concerned here with detailed mechanical theories, but remark that there are two experimentally well documented types of kinematical change (i. e. change of crystal state) which are generally reckoned to play a significant role in any mechanical theory which purports to describe these materials. The first of these types of change of state is elastic deformation: two crystal states, $\Sigma$ and $\Sigma^{\prime} \equiv\left\{\ell_{a}^{\prime}(\cdot), \Omega^{\prime} ; a=1,2,3\right\}$ are said to be elastically related to one another if there exists a smooth invertible mapping $\boldsymbol{u}: \Omega \rightarrow \boldsymbol{u}(\Omega)=\Omega^{\prime}$ such that

$$
\ell_{a}^{\prime}(\boldsymbol{u}(\boldsymbol{x}))=\nabla \boldsymbol{u}(\boldsymbol{x}) \ell_{a}(\boldsymbol{x}), \quad a=1,2,3 .
$$

It is commonly held that a change of state which is inelastic (i. e., not an elastic deformation) is irreversible, in a thermodynamic sense. (We do not embrace that prejudice here). The second type is a particular inelastic change of state: in slip, or rearrangement of the lattice vector fields, thin layers of the crystal slide one over the other with the 'internal structure' (i.e. the distribution of fields in each layer) unchanged in the process. We shall not need to be mathematically precise regarding this type of change of state in this paper (see Chipot and Kinderlehrer [4], Fonseca and Parry [9] for such treatments).

We proceed as follows: in the next section we motivate the consideration of symmetry in defective crystals by reviewing results for a variational problem that corresponds to minimizing a functional which represents the energy of a perfect crystal (in that the corresponding energy density has perfect crystal symmetries). It turns out that the symmetry properties determine qualitative properties of the set of minimizers. Subsequently, we indicate how 'elastic invariants' may be constructed from the lattice vector fields and their derivatives - the invariants are measures of inelastic change (since they are invariant under elastic deformation). It turns out that certain rearrangements of the lattice vector fields also preserve the elastic invariants, so that the 'slip' type of change of state has an abstract status in this kinematical theory, subordinate to the elastic invariants.

The main part of the paper describes how one associates a discrete structure with the continuous lattice vector fields of (1.1), how a simple constitutive assumption regarding the energy density of a defective crystal leads to the theory of Lie groups and their discrete subgroups, how perfect crystal symmetries are generalized to the defective crystal case. It will turn out that the symmetries with which we are concerned correspond to changes in sets of generators of certain discrete Lie groups. We indicate, briefly, that particular symmetries (those which extend to automorphisms of the corresponding discrete group) extend to symmetries of the continuous model (the automorphisms of the ambient Lie group), following Gorbatsevich [10] in part. This last observation is important from the continuum mechanics point of view, for it shows that certain discrete symmetries are embedded in elastic deformation, so 
that these discrete geometrical changes can themselves be regarded as elastic, even though elastic deformation is a continuum concept.

\section{A VARIATIONAL PROBLEM FOR PERFECT CRYSTALS}

In the context of elastic changes of state determined by a deformation gradient $F(x) \equiv \nabla \boldsymbol{u}(\boldsymbol{x})$, cf. (1.3), Chipot and Kinderlehrer [4] considered the following variational problem: for a given non-singular $3 \times 3$ matrix $A$, find

where

$$
E_{\Omega}(A) \equiv \inf _{\mathcal{A}(A)} \int_{\Omega} w(\nabla \boldsymbol{u}(\boldsymbol{x})) d \boldsymbol{x},
$$

$$
\mathcal{A}(A) \equiv\left\{\boldsymbol{u} \in W^{1, \infty}\left(\Omega ; \mathbb{R}^{3}\right) ; \operatorname{det} \nabla \boldsymbol{u}>0 \text { a.e. in } \Omega, \boldsymbol{u}=A \boldsymbol{x} \text { on } \partial \Omega\right\},
$$

and where the prescribed energy density function $w$ has the properties

$$
w(F) \geq 0, \quad w(\mathbb{I})=0, \quad \lim _{\operatorname{det} F \rightarrow 0} w(F)=\infty, \quad w(F)=w(Q F H),
$$

whenever $Q$ is proper orthogonal, $\operatorname{det}(F)>0$, and $H$ is an element of a particular group conjugate to $\mathrm{GL}_{3}(\mathbb{Z})$. The requirement on the form of the matrix $H$ relates to the assumption that the material under consideration is a perfect crystal - it derives from two facts: first that if $\boldsymbol{e}_{1}, \boldsymbol{e}_{2}, \boldsymbol{e}_{3}$ is an integral basis of $L$, cf. (1.2), then $\boldsymbol{e}_{1}^{\prime}, \boldsymbol{e}_{2}^{\prime}, \boldsymbol{e}_{3}^{\prime}$ is also a basis of $L$ if and only if $\boldsymbol{e}_{a}^{\prime}=\gamma_{a b} \boldsymbol{e}_{b}, a, b=1,2,3$, where $\gamma \equiv\left(\gamma_{a b}\right) \in$ $\mathrm{GL}_{3}(\mathbb{Z})$; second that the crystal structure corresponding to deformation gradient $F$ is a perfect lattice with basis vectors $F e_{1}, F e_{2}, F e_{3}$. They find that:

(i) $E_{\Omega}(A)=|\Omega| \widetilde{w}(A)$ whenever $\Omega \subset \mathbb{R}^{3}$ is a bounded domain with $\partial \Omega$ of measure zero;

(ii) $\tilde{w}$ is the greatest convex function less that the function $\phi$ defined by

$$
\phi(\operatorname{det} A)=\inf _{\operatorname{det} F=\operatorname{det} A} w(F) ;
$$

(iii) For all uniformly bounded (in $L^{\infty}$ ) minimizing sequences $\left\{\nabla \boldsymbol{u}_{n}\right\}$ of (2.1), with corresponding Young measure $\left\{\mu_{\boldsymbol{x}}\right\}_{\boldsymbol{x} \in \Omega}$, the average limiting Cauchy stress, defined by

$$
\bar{T}(\boldsymbol{x})=\int_{M^{3 \times 3}} \frac{1}{\operatorname{det} F} \frac{\partial w}{\partial F}(F) F^{T} d \mu_{\boldsymbol{x}},
$$

where $F^{T}$ denote the transpose of $F, M^{3 \times 3}$ is the set of all $3 \times 3$ matrices, is a pressure, i. e., $\bar{T}(x)=\alpha(x) \mathbb{I}$, for some $\alpha: \mathbb{R}^{3} \rightarrow \mathbb{R}, \mathbb{I}$ the $3 \times 3$ identity.

In particular, according to (iii) the 'perfect' symmetry of the energy density, expressed through the requirement $w(F)=w(Q F H)$, cf. (2.3), implies that the crystal cannot sustain any stress other than a pressure i. e., it cannot sustain shear stresses in equilibrium. This result is contradicted by experiment, so one is forced to concede that real crystals are not perfect, and one is forced to consider what symmetry properties should apply to energy density functions appropriate for defective crystals. 
Note that Chipot and Kinderlehrer's results were extended by Fonseca and Parry [9] to the case where the class of competitor functions $\mathcal{A}(A)$ includes rearrangements of the crystal state as well as elastic deformations.

\section{INVARIANT INTEGRALS}

Since inelastic changes of state play an essential role in the mechanics of defective crystals, it is important to consider the following question: given two crystal states, how does one determine whether or not those states are elastically related to one another. To discuss this we construct elastic invariants, which are unchanged in elastic deformation - then if two states are elastically related, it is a necessary condition that these objects match in the two states. As an example, consider the scalar fields

$$
S_{a b} \equiv \nabla \wedge \boldsymbol{d}_{a} \cdot \boldsymbol{d}_{b} / \operatorname{det}\left(\left\{\boldsymbol{d}_{a}\right\}\right), \quad a, b=1,2,3,
$$

where $d_{1}(\cdot), d_{2}(\cdot), d_{3}(\cdot)$ are fields dual to the lattice vector fields, and

$$
\operatorname{det}\left(\left\{\boldsymbol{d}_{a}\right\}\right) \equiv \boldsymbol{d}_{1} \cdot \boldsymbol{d}_{2} \wedge \boldsymbol{d}_{3}
$$

One calculates that if (1.3) holds, and if $S_{a b}^{\prime}$ is calculated from the fields $\ell_{a}^{\prime}(\cdot), a=$ $1,2,3$, then

$$
S_{a b}^{\prime}(\boldsymbol{u}(\boldsymbol{x}))=S_{a b}(\boldsymbol{x}), \quad a, b=1,2,3 .
$$

Therefore

$$
\underset{\boldsymbol{y} \in \Omega^{\prime}}{\operatorname{range}} S_{a b}^{\prime}(\boldsymbol{y})=\underset{\boldsymbol{x} \in \Omega}{\operatorname{range}} S_{a b}(\boldsymbol{x}), \quad a, b=1,2,3,
$$

and each of the nine quantities range $\boldsymbol{x}_{\boldsymbol{x} \in \Omega} S_{a b}(\boldsymbol{x})$ is an elastic invariant. Likewise, for example, if $\zeta$ is a circuit in $\Omega$, one calculates that $\oint_{\zeta} \boldsymbol{d}_{a} \cdot \boldsymbol{d} \boldsymbol{x}, a=1,2,3$, is an elastic invariant integral. In fact, there exists an infinite number of elastic invariant objects, there is a finite functional basis for these quantities, see Olver [16], Davini and Parry $[6,7]$. So one obtains a finite number of conditions necessary that two crystal states be elastically related.

Consider the particular case where $\Sigma=\left\{\boldsymbol{e}_{a}, \Omega ; a=1,2,3\right\}$, where $\boldsymbol{e}_{1}, \boldsymbol{e}_{2}, \boldsymbol{e}_{3}$ is a basis of $\mathbb{R}^{3}$ with $\boldsymbol{e}_{1} \cdot \boldsymbol{e}_{2} \wedge \boldsymbol{e}_{3}=1$, so that the lattice vector fields are constant in $\Omega$. Then all of the quantities $S_{a b}, a, b=1,2,3$, are zero, and it turns out that $\Sigma^{\prime} \equiv\left\{\ell_{a}^{\prime}(\cdot), \Omega\right\}$ (we choose $\Omega^{\prime}=\Omega$ for simplicity) is such that all the elastically invariant objects match those of $\Sigma$ if and only if

$$
d_{a}^{\prime}(x)=\nabla \tau_{a}(x), x \in \Omega, a=1,2,3 ; d_{1}(x) \cdot d_{2}(x) \wedge d_{3}(x)=1, x \in \Omega,
$$

for some potentials $\tau_{1}, \tau_{2}, \tau_{3}$ such that $\nabla \tau_{1}, \nabla \tau_{2}, \nabla \tau_{3}$ are linearly independent at each $x \in \Omega$. The important point to note is that $\Sigma$ and $\Sigma^{\prime}$ are not elastically related to each other, in this case. Indeed, $\Sigma^{\prime}$ is elastically related to $\widetilde{\Sigma} \equiv\left\{\boldsymbol{e}_{a}, \boldsymbol{\tau}(\Omega)\right\}$ where $\tau \equiv\left(\tau_{a}\right)$, but $\tilde{\Sigma}$ is not elastically related to $\Sigma$ because, if that were so, there would exist $\boldsymbol{u}: \Omega \rightarrow \boldsymbol{u}(\Omega)=\boldsymbol{\tau}(\Omega)$ such that $\nabla \boldsymbol{u}=\mathbb{I}$, from (1.3). Then we would have that $\boldsymbol{u}=\boldsymbol{x}+\boldsymbol{c}, \boldsymbol{c} \in \mathbb{R}^{3}, \boldsymbol{u}(\Omega)=\boldsymbol{\tau}(\Omega)=\Omega+\boldsymbol{c}$, which is false for general choices of $\tau$. One says that $\Sigma$ and $\tilde{\Sigma}$ are related by slip, or rearrangement of the lattice vector 
fields, since $\Sigma$ and $\tilde{\Sigma}$ are defined on regions which have the same volume (because $\operatorname{det} \nabla \boldsymbol{\tau}=1$ in $\Omega$ ), and the corresponding fields $\ell_{a}(\cdot), \tilde{\ell}_{a}(\cdot)$ are restrictions of fields which have the same constant values in $\mathbb{R}^{3}$. (This is just a particular example of slip).

The slip mechanism is the archetypical inelastic change of state in phenomenological theories of defective crystals. The remarks above that indicate the slips are inelastic changes of state which preserve the elastic invariants - and this is so.

\section{ENERGY DENSITY FOR DEFECTIVE CRYSTALS, SYMMETRY OF ENERGY} DENSITY

We assume that point values of quantities associated with the lattice vector fields determine the values of a corresponding energy density function $w$, and we make what seems to be the simplest extrapolation of elasticity theory that accounts for the existence of continuous distributions of defects. We assume that

$$
w=w\left(\left\{\ell_{a}\right\}, S\right) .
$$

In (4.1) $\left\{\ell_{a}\right\}$ denotes a set of vectors $\ell_{1}, \ell_{2}, \ell_{3} \in \mathbb{R}^{3}$, assumed to consist of values of the fields $\ell_{1}(\cdot), \ell_{2}(\cdot), \ell_{3}(\cdot)$ at some point, the origin $\mathbf{0}$ say, and $S$ lies in a particular class of $3 \times 3$ matrices. The field $S(\cdot) \equiv\left(S_{a b}(\cdot)\right)$, defined through the duals of the lattice vector fields by (3.1), measures the non-commutativity of the fields $\left\{\ell_{a}(\cdot)\right\}$ to some extent, so it is nonzero in the case of a defective crystal, by definition. $S(\cdot)$ is called the dislocation density tensor (even though it represents nine scalar fields). As an argument of the energy density $w$, we are given just one value of the field $S(\cdot)$ and no information regarding any of the derivatives of $S(\cdot)$ - so we make the major assumption that the field $S(\cdot)$ is constant in $\Omega$. The fact that $S(\cdot)$ is constant restricts the possible values that the constant $S=S(\mathbf{0})$, say, may take, but we do not make this explicit.

In a different language, the arguments of the energy density are determined by a 'uniform crystal state,' which is a crystal state where the lattice vector fields have constant dislocation density $S$, and $\ell_{a}(\mathbf{0})=\ell_{a}, a=1,2,3$.

In the particular case where $S=0$, so the crystal is 'perfect', it is a traditional a priori assumption that the symmetries of the energy density $w\left(\left\{\ell_{a}\right\}, 0\right)$ correspond to symmetries of the perfect lattice $L$ with integral basis vectors $\ell_{1}, \ell_{2}, \ell_{3}$. Thus the symmetries of the continuum energy density are derived from an associated discrete structure, $L$ in this case. We shall consider, in the following sections, ways of associating a discrete structure, $D$, say, with given arguments $\left\{\ell_{a}\right\}, S$ of the energy density, in the general case where $S \neq 0$. Also, we shall take the point of view that if different arguments $\left(\left\{\ell_{a}^{\prime}\right\}, S^{\prime}\right)$ lead to the same discrete structure $D$, then

$$
w\left(\left\{\ell_{a}\right\}, S\right)=w\left(\left\{\ell_{a}^{\prime}\right\}, S^{\prime}\right),
$$

and say that such equivalent arguments, $\left\{\ell_{a}\right\}, S$ and $\left\{\ell_{a}^{\prime}\right\}, S^{\prime}$, represent 'symmetries' of the energy density (this argument mimics what is done when $S=0$ ). We shall also 
be concerned with whether or not such equivalent arguments correspond to uniform crystal states which are elastically related to one another, for in the case when such arguments are so related there will be a precise sense in which particular discrete symmetries of (4.2) represent elastic changes of state.

\section{Defective analogues of PeRfect lattices AND Links to the theory OF LIE GROUPS}

We are interested in uniform defective crystal configurations where the lattice vector fields $\left\{\ell_{a}(\cdot)\right\}$ are defined in $\Omega \equiv \mathbb{R}^{3}$, and are such that the dislocation density tensor $S(\cdot)$ is constant in $\mathbb{R}^{3}$. The condition that $S$ is constant is an integrability condition which guarantees that for given lattice vector fields, the partial differential system

$$
\ell_{a}(\boldsymbol{\psi}(\boldsymbol{x}, \boldsymbol{y}))=\nabla_{1} \boldsymbol{\psi}(\boldsymbol{x}, \boldsymbol{y}) \ell_{a}(\boldsymbol{x}), \quad a=1,2,3,
$$

where $\nabla_{1} \psi(\cdot, \cdot)$ denotes the gradient of $\psi$ with respect to its first argument, has a solution for the function $\psi$. Moreover, the function $\psi: \mathbb{R}^{3} \times \mathbb{R}^{3} \rightarrow \mathbb{R}^{3}$ can be taken to satisfy the properties required for it to be a Lie group composition function, i. e.

$$
\begin{gathered}
\psi(\mathbf{0}, x)=\psi(x, 0)=x, \\
\psi\left(x, x^{-1}\right)=\psi\left(x^{-1}, x\right)=0, \\
\psi(\psi(x, y), z)=\psi(x, \psi(y, z)),
\end{gathered}
$$

where $\mathbf{0}$ is the group identity element and $\boldsymbol{x}^{-1}$ is the unique inverse of the element $\boldsymbol{x}[17,21]$. Given an appropriate value of the dislocation density tensor $S$ one can specify a corresponding Lie group $G$ by constructing fields $\ell_{a}(\cdot), a=1,2,3$ such that the dual fields satisfy (3.1) and then solving (5.1) for the group composition function $\psi$.

Since the dislocation density tensor $S$ is an elastic invariant, the composition function $\psi$ obtained from (5.1) is just one amongst the infinite number of those which may be found by making different choices of the lattice vector fields, given a value of $S$.

Each Lie group $G=\left(\mathbb{R}^{3}, \boldsymbol{\psi}\right)$ has a corresponding Lie algebra $\mathfrak{g}$ consisting of the vector space $\mathbb{R}^{3}$, here, with Lie bracket operation $[\cdot, \cdot]: \mathbb{R}^{3} \times \mathbb{R}^{3} \rightarrow \mathbb{R}^{3}$ defined by

$$
[\boldsymbol{x}, \boldsymbol{y}]=C_{i j k} x_{j} y_{k} \boldsymbol{e}_{i}, \quad \boldsymbol{x}, \boldsymbol{y} \in \mathbb{R}^{3},
$$

where $\left\{\boldsymbol{e}_{1}, \boldsymbol{e}_{2}, \boldsymbol{e}_{3}\right\}$ is a basis of $\mathbb{R}^{3}, \boldsymbol{x}=x_{j} \boldsymbol{e}_{j}, \boldsymbol{y}=y_{j} \boldsymbol{e}_{j}, C_{i j k}$ are the structure constants given by

$$
C_{i j k}=\frac{\partial^{2} \psi_{i}}{\partial x_{j} \partial y_{k}}(\mathbf{0}, \mathbf{0})-\frac{\partial^{2} \psi_{i}}{\partial x_{k} \partial y_{j}}(\mathbf{0}, \mathbf{0}),
$$

where $\boldsymbol{\psi}(\boldsymbol{x}, \boldsymbol{y})=\psi_{i}(\boldsymbol{x}, \boldsymbol{y}) \boldsymbol{e}_{i}$. The structure constants with respect to the basis $\left\{\boldsymbol{e}_{1}, \boldsymbol{e}_{2}, \boldsymbol{e}_{3}\right\}$ are related to the components of the dislocation density tensor by

$$
C_{k i j} \ell_{r j}(\mathbf{0}) \ell_{s k}(\mathbf{0})=\epsilon_{p r s} S_{k p} \ell_{k i}(\mathbf{0}),
$$


where $\epsilon_{r i j}$ is the permutation symbol and $\ell_{r}(\mathbf{0})=\ell_{r j}(\mathbf{0}) e_{j}$, see Elzanowski and Parry [8].

Given a value of $S$, for a particular choice of $\psi$ (equivalently $G$ ), the corresponding geometrical structure $D$, which we take to be the defective crystal analogue of the perfect lattice, is constructed as follows. Let $\ell_{a}(\cdot), a=1,2,3$, satisfy (5.1) and let $v_{1}, v_{2}, v_{3}$ be given real numbers. Define the integral curve through $\boldsymbol{x}_{0}$ of the field $v_{a} \ell_{a}(\cdot)$ to be the solution $\{x(t): t \in \mathbb{R}\}$ of the ordinary differential equation $\dot{\boldsymbol{x}}(t)=v_{a} \ell_{a}(\boldsymbol{x}(t)), \boldsymbol{x}(0)=\boldsymbol{x}_{0}$. Note that $\boldsymbol{v}:=v_{a} \ell_{a}(\mathbf{0})$ determines the field $v_{a} \ell_{a}(\cdot)$ by (5.1). One defines the mapping $\exp (v): G \rightarrow G$, and the group element $\mathrm{e}^{(\boldsymbol{v})}$, by

$$
\exp (\boldsymbol{v})\left(x_{0}\right)=x(1), \mathrm{e}^{(\boldsymbol{v})}=\exp (\boldsymbol{v})(\mathbf{0})
$$

It is standard result of Lie group theory that

$$
\exp (\boldsymbol{v})(\boldsymbol{x})=\boldsymbol{\psi}\left(\mathrm{e}^{(\boldsymbol{v})}, \boldsymbol{x}\right),
$$

which states that the flow along the integral curves of the lattice vector fields corresponds to group multiplication by the group element $\mathrm{e}^{(\boldsymbol{v})}$.

The set of points of the geometrical structure $D$ can be built through an iteration process. One begins with the origin then adds the six points which are reached by flowing along each of the vector fields $\ell_{a}(\cdot), a=1,2,3$, forwards and backwards one 'unit', i.e. $\mathrm{e}^{\left(\ell_{a}\right)}$ and $\mathrm{e}^{-\left(\ell_{a}\right)}, a=1,2,3$, where $\ell_{a}=\ell_{a}(\mathbf{0})$. One repeats this process starting at each of these six points, and continues in this way. By (5.6) the structure, $D$ that one obtains in this way is the subgroup of $G$ that is generated by the group elements $\mathrm{e}^{\left(\ell_{1}\right)}, \mathrm{e}^{\left(\ell_{2}\right)}, \mathrm{e}^{\left(\ell_{3}\right)}$ (noting that $\mathrm{e}^{-\left(\ell_{a}\right)}$ is the inverse of $\mathrm{e}^{\left(\ell_{a}\right)}$ in the group $G$ ).

Remark 1. In the case of perfect crystals $S=0$, and one can choose $\ell_{a}(\boldsymbol{x}) \equiv$ $\ell_{a}(\mathbf{0}) \equiv \ell_{a}$ as a solution of (3.1) where $\left\{\ell_{1}, \ell_{2}, \ell_{3}\right\}$ is a basis of $\mathbb{R}^{3}$. Then $\boldsymbol{\psi}(\boldsymbol{x}, \boldsymbol{y})=$ $\boldsymbol{x}+\boldsymbol{y}$ is a solution of (5.1) which has the properties of a Lie group composition function. Flow along the lattice vector fields corresponds in this case to translation by $\ell_{1}, \ell_{2}, \ell_{3}$. Successive translations produce the perfect lattice $L$ as in (1.2). Hence the geometrical structure $D$ produced by the iteration process described above is a generalisation of the perfect lattice $L$.

Let $G$ be a three-dimensional Lie group with group multiplication $\psi$ and write $\boldsymbol{\psi}(\boldsymbol{x}, \boldsymbol{y}) \equiv \boldsymbol{x} \boldsymbol{y}$ as an alternative notation. Let $(\boldsymbol{x}, \boldsymbol{y})=\boldsymbol{x}^{-1} \boldsymbol{y}^{-1} \boldsymbol{x}, \boldsymbol{y}$ denote the commutator of two group elements $x, y \in G$ and denote by $G^{\prime}$ the commutator subgroup of $G$ - that is, the group generated by all commutators of elements of $G$. Notice that $\left(\mathrm{e}^{\left(\ell_{a}\right)}, \mathrm{e}^{\left(\ell_{b}\right)}\right)=\mathrm{e}^{-\left(\ell_{a}\right)} \mathrm{e}^{-\left(\ell_{b}\right)} \mathrm{e}^{\left(\ell_{a}\right)} \mathrm{e}^{\left(\ell_{b}\right)}$ is the group element obtained by successive flows along the vector fields $\ell_{b}(\cdot), \ell_{a}(\cdot),-\ell_{b}(\cdot),-\ell_{a}(\cdot)$ so there is a clear correspondence between the commutator of group elements $\mathrm{e}^{\left(\ell_{a}\right)}, \mathrm{e}^{\left(\ell_{b}\right)}$ and the construction of the Burgers vector in mechanics. 


\subsection{Conditions for discreteness}

In general, the geometric structures generated by the iterative process outlined above are not discrete sets of points. The structures $D$ are discrete when $D$ is a uniform discrete subgroup of the Lie group $G$ (i. e. the left coset space $G / D$ is compact). This is a generalisation of the requirement in the perfect crystal case that $\mathbb{R}^{3} / L$ (the unit cell of the lattice $L$ with appropriate identification of boundary points) is compact. According to Auslander et al. [1] there are three classes of three-dimensional Lie group which contain uniform discrete subgroups. These are a certain class of nilpotent groups and two non-isomorphic classes of solvable Lie groups. Recall that a three-dimensional Lie group $G$ is nilpotent if $\left(G^{\prime}, G\right)=\mathbf{0}$, i. e. elements of the commutator subgroup $G^{\prime}$ commute with elements of the group $G$. Also a threedimensional Lie group $G$ is solvable if $\left(G^{\prime}, G^{\prime}\right)=\mathbf{0}$, i. e. elements of the commutator subgroup commute with each other.

We next summarize conditions on the dislocation density tensor which guarantee that the structures $D$ are discrete, and give the forms of $D$ explicitly, in the cases $D$ is a uniform discrete subgroup of a three-dimensional nilpotent or (one type of) solvable Lie group. We also discuss the symmetries of $D$.

\section{THE NILPOTENT CASE}

Suppose that $G$ is a three-dimensional nilpotent Lie group which contains a uniform discrete subgroup $D$. Mal'cev [12] shows that $D$ must be generated by three elements and that the Lie algebra $\mathfrak{g}$ corresponding to $G$ must have rational structure constants with respect to some basis. Taking an equivalent approach, following Thurston [22], it can be shown, by assuming first that there is a non-zero minimum distance between the points of $D$ (generated by the iterative process outlined in section 2) and second that the points $\mathrm{e}^{\left(\ell_{a}\right)}, a=1,2,3$, are 'close enough' to the origin (see Parry [17] for details) that the Lie group $G$ must be nilpotent and that the dislocation density has the form

$$
S_{a b}=\lambda v_{a} v_{b} \quad a, b=1,2,3,
$$

where $\lambda \in \mathbb{Q}$, and $v_{1}, v_{2}, v_{3}$ are relatively prime integers [3,17].

Now recall that, given a value of the dislocation density tensor $S$ of the form (6.1), there are infinitely many possible choice of corresponding nilpotent Lie groups $G$ corresponding to different choices of $\boldsymbol{\psi}$. We make a canonical choice as follows. Let $\psi$ be the group composition function for a three dimensional nilpotent Lie group $G$ and let $\mathrm{e}^{(\cdot)}: \mathfrak{g} \rightarrow G$ be the exponential function as defined by (5.5). The CampbellBaker-Hausdorff formula gives an explicit expression for $c$ in the relation $\mathrm{e}^{(\boldsymbol{c})}=$ $\mathrm{e}^{(\boldsymbol{a})} \mathrm{e}^{(\boldsymbol{b})}$ for $\boldsymbol{a}, \boldsymbol{b} \in \mathfrak{g}$. For three-dimensional nilpotent Lie groups

$$
\boldsymbol{c}=\boldsymbol{a}+\boldsymbol{b}+\frac{1}{2}[\boldsymbol{a}, \boldsymbol{b}],
$$

and $\boldsymbol{c}(\boldsymbol{a}, \boldsymbol{b})$ satisfies the required conditions for it to be a group composition function on $\mathbb{R}^{3}$. Thus for any $G$ in the relevant isomorphism class of Lie groups one can 
define a canonical Lie group $J$ by taking the group operation in $J$ to be given by $\boldsymbol{\psi}^{\prime}(\boldsymbol{a}, \boldsymbol{b})=\boldsymbol{c}$. With this choice of composition function the one parameter subgroups of $J$ are straight lines through the origin and $\mathrm{e}^{(\boldsymbol{x})}=\boldsymbol{x}$ (Lie group and Lie algebra elements can be identified).

When the components of the dislocation density tensor have the form (6.1) the structure constants with respect to the basis $\boldsymbol{e}_{1}, \boldsymbol{e}_{2}, \boldsymbol{e}_{3}$ where $\boldsymbol{e}_{a}=\ell_{a}(\mathbf{0})$, are given by

$$
C_{i j k}=\lambda v_{i} v_{r} \epsilon_{r j k} \text {. }
$$

Then the composition function in $J$ has the form (dropping the prime in $\boldsymbol{\psi}^{\prime}$ )

$$
\psi(x, y)=x+y+\frac{1}{2} \lambda v(v \cdot x \wedge y) \quad x, y \in \mathbb{R}^{3},
$$

where $\boldsymbol{v}=v_{r} \boldsymbol{e}_{r}$.

Let $D$ be the discrete subgroup of $J$ which is generated by $\mathrm{e}^{\left(\ell_{1}\right)}, \mathrm{e}^{\left(\ell_{2}\right)}, \mathrm{e}^{\left(\ell_{3}\right)}$. The translation group $T$ of $D$ is defined by

$$
T=\{\boldsymbol{t} \in J: \text { if } \boldsymbol{d} \in D, \boldsymbol{d}+\boldsymbol{t} \in D\} .
$$

Let $\lambda=p / q \in \mathbb{Q}$ where $p$ and $q \in \mathbb{Z}$ have no common factors and define $v=$ $v_{1} v_{2} v_{3}$. Also define the integer $k$ as

$$
k= \begin{cases}p & \text { if } v \text { is even or if ( } v \text { is odd and } p \in 4 \mathbb{Z} \text { ) } \\ \frac{p}{2} & \text { if } v \text { is odd and } p \in 2 \mathbb{Z}, p \notin 4 \mathbb{Z} \\ 2 p & \text { if } v \text { is odd and } p \text { is odd. }\end{cases}
$$

Cermelli and Parry [3] show that if $k$ is even then $T=D$ and $T$ consists of all integer linear combinations of $\boldsymbol{e}_{1}, \boldsymbol{e}_{2}, \boldsymbol{e}_{3}, \lambda \boldsymbol{v} / k$. Thus the points of $D$ form a three-dimensional lattice, as an integral basis of $T=D$ may be found in terms of $\boldsymbol{e}_{1}, \boldsymbol{e}_{2}, \boldsymbol{e}_{3}, \lambda \boldsymbol{v} / k$. Also, if $k$ is odd they show that $T$ consists of all integer linear combinations of $2 e_{1}, 2 e_{2}, 2 e_{3}, \lambda v / k$ and $D / T$ has four elements which may be written as $T, \boldsymbol{\alpha} T, \boldsymbol{\beta} T, \boldsymbol{\alpha} \boldsymbol{\beta} T$ for some $\boldsymbol{\alpha}, \boldsymbol{\beta} \in D$. Thus the points of $D$ form a 4-lattice in the sense of Pitteri and Zanzotto [20].

\subsection{Canonical Coordinates and Symmetries of D}

The uniform discrete subgroup $D \subset J$ is generated by three elements $\mathrm{e}^{\left(\ell_{1}\right)}, \mathrm{e}^{\left(\ell_{2}\right)}$, $\mathrm{e}^{\left(\ell_{3}\right)}$. According to Mal'cev [12], in a three dimensional nilpotent Lie group with structure constants given by (6.3) there are generators of $D$ and corresponding Lie algebra elements $c_{1}, c_{2}, c_{3} \in \mathbb{R}^{3}$ such that for some integer $k_{c}$

$$
\left[c_{1}, c_{2}\right]=k_{c} c_{3}, \quad\left[c_{1}, c_{3}\right]=\left[c_{2}, c_{3}\right]=\mathbf{0} .
$$

Recalling that group and algebra elements may be identified in $J$, Mal'cev shows further that any $d \in D$ can be written uniquely in the form

$$
\boldsymbol{d}=c_{1}^{\alpha} c_{2}^{\beta} c_{3}^{\gamma}, \quad \alpha, \beta, \gamma \in \mathbb{Z} .
$$


(This relation is a generalisation of the expression of a point $\boldsymbol{x}$ of a perfect lattice with basis $\ell_{1}, \ell_{2}, \ell_{3}$ as $\boldsymbol{x}=\alpha \ell_{1}+\beta \ell_{2}+\gamma \ell_{3}, \alpha, \beta, \gamma \in \mathbb{Z}$.) Also note that, for $\boldsymbol{x}, \boldsymbol{y} \in J, \boldsymbol{x}^{-1}=-\boldsymbol{x}$, etc., and one can show that the commutator and Lie bracket can also be identified. In particular,

$$
\left(c_{1}, c_{2}\right)=c_{3}^{k_{c}}, \quad\left(c_{1}, c_{3}\right)=\left(c_{2}, c_{3}\right)=\mathbf{0},
$$

so the commutator subgroup $D^{\prime}$ is generated by $c_{3}^{k_{c}}$. The commutator $\left(c_{1}, c_{2}\right)=c_{3}^{k_{c}}$ represents flow along the vector fields defined by the Lie algebra elements $c_{2}, c_{1},-c_{2},-c_{1}$ successively, because Lie group and algebra elements can be identified in this canonical representation. The corresponding Burgers vector is an integer multiple of the third vector $c_{3}$ and one may picture this lack of commutativity as a 'screw dislocation'.

Let the discrete subgroup $D \subset J$ be generated by $c_{1}, c_{2}, c_{3}$ where these three elements provide a canonical basis for $D$ as described above. Parry and Sigrist [19] compute the necessary and sufficient conditions that elements $\boldsymbol{e}_{1}, \boldsymbol{e}_{2}, \boldsymbol{e}_{3} \in D$ also generate $D$. These changes of generators are the geometrical symmetries of the set of points $D$. The computations in [19] use the fact that the translation group consists of all integer linear combinations of $c_{1}, c_{2}, c_{3}$ in the case that $k_{c}$ is even and consists of all integer linear combinations of $2 c_{1}, 2 c_{2}$ and $c_{3}$ in the case that $k_{c}$ is odd.

Let $D_{\boldsymbol{e}}$ denote the group generated by $\boldsymbol{e}_{1}, \boldsymbol{e}_{2}, \boldsymbol{e}_{3} \in D$. In the case where the (canonical) generators of $D$ satisfy $\left(c_{1}, c_{2}\right)=c_{3}^{k_{c}}$ with $k_{c}$ even there exists a matrix $\gamma$ of integers with determinant $\Gamma$, say, such that $\boldsymbol{e}_{i}=\gamma_{i j} \boldsymbol{c}_{j}$ and $\gamma^{-1}= \pm \frac{1}{\Gamma}\left(p_{l k}\right)$ where $p_{l k} \in \mathbb{Z}$. We define $v_{i}=p_{3 i}$. (There are similar results in the case $k_{c}$ odd). Parry and Sigrist [19] prove the following result regarding the geometrical symmetries of $D$ (we give the results only for the case $k_{c}$ even).

Theorem 2. $D_{e}=D$ if and only if there exist integers $l_{0}, m_{0}, \Gamma$, and a matrix $A \in \mathrm{GL}_{3}(\mathbb{Z})$ with third row of relatively prime integers $v_{1}, v_{2}, v_{3}$ with $v:=v_{1} v_{2} v_{3}$ such that if $k_{c}$ is even, then

$$
\left(\begin{array}{ccc}
1 & 0 & -l_{0} \\
0 & 1 & -m_{0} \\
0 & 0 & \pm \Gamma
\end{array}\right)\left(\begin{array}{l}
c_{1} \\
c_{2} \\
c_{3}
\end{array}\right)=A\left(\begin{array}{l}
e_{1} \\
e_{2} \\
e_{3}
\end{array}\right)
$$

where if $k_{c} \in 4 \mathbb{Z}$ then $h c f\left(k_{c}, \Gamma\right)=1$ or if $k_{c} \in 2 \mathbb{Z}, k_{c} \notin 4 \mathbb{Z}$ then either ( $v$ is even and $\left.h c f\left(k_{c}, \Gamma\right)=1\right)$ or (v is odd and $h c f\left(k_{c}, \Gamma\right)=2$ ).

\section{The SOlvable CASES}

According to Auslander et al [1] there are two non-isomorphic classes of threedimensional solvable Lie groups which have uniform discrete subgroups. They call these two classes $S_{1}$ and $S_{2}$. Group elements $\boldsymbol{x} \in S_{p}, p=1,2$, are identified with points of $\mathbb{R}^{3}$ by expressing them as $\boldsymbol{x}=x_{i} \boldsymbol{e}_{i}$ with respect to some basis $\boldsymbol{e}_{1}, \boldsymbol{e}_{2}, \boldsymbol{e}_{3}$. Auslander et al. [1] choose to represent the group elements as $4 \times 4$ matrices (still 
parameterised by $\left.\left(x_{1}, x_{2}, x_{3}\right)\right)$ and these matrix representations form an isomorphic group $S_{p, m}$ where the matrix representation of $\boldsymbol{x} \in S_{p}$ is $r_{m}(\boldsymbol{x}) \in S_{p, m}$ which has the form

$$
r_{m}(\boldsymbol{x}) \equiv\left(\begin{array}{cc|cc}
\phi\left(x_{3}\right) & 0 & x_{1} \\
& 0 & x_{2} \\
\hline 0 & 0 & 1 & x_{3} \\
0 & 0 & 0 & 1
\end{array}\right), \boldsymbol{x} \equiv\left(\begin{array}{l}
x_{1} \\
x_{2} \\
x_{3}
\end{array}\right) \in \mathbb{R}^{3}, \phi\left(x_{3}\right)=\left(\begin{array}{ll}
a\left(x_{3}\right) & b\left(x_{3}\right) \\
c\left(x_{3}\right) & d\left(x_{3}\right)
\end{array}\right) .
$$

Here, $\left\{\phi\left(x_{3}\right): x_{3} \in \mathbb{R}\right\}$ is a one parameter subgroup of the unimodular group $\mathrm{SL}_{2}(\mathbb{R})$ and $\phi(1) \in \mathrm{SL}_{2}(\mathbb{Z})$. Let us define

$$
\phi(1) \equiv \theta=\left(\begin{array}{ll}
a(1) & b(1) \\
c(1) & d(1)
\end{array}\right)=\left(\begin{array}{ll}
a & b \\
c & d
\end{array}\right), \quad a, b, c, d \in \mathbb{Z}, \quad a d-b c=1 .
$$

If $\operatorname{tr}(\theta)>2$ then the eigenvalues of $\theta$ are real and distinct and the group of matrices of the form $r_{m}(\boldsymbol{x})$ is isomorphic to $S_{1}$. We discuss $S_{1}$ only. ( $S_{2}$ will be treated elsewhere). Group multiplication $\psi$ in $S_{1}$ is defined as follows:

$$
r_{m}(\boldsymbol{x}) r_{m}(\boldsymbol{y})=r_{m}(\boldsymbol{\psi}(\boldsymbol{x}, \boldsymbol{y})), \quad \boldsymbol{x}, \boldsymbol{y} \in \mathbb{R}^{3}
$$

Let us also define

$$
\phi^{\prime}(0)=\mathcal{A}=\left(\begin{array}{ll}
a^{\prime}(0) & b^{\prime}(0) \\
c^{\prime}(0) & d^{\prime}(0)
\end{array}\right), \quad \text { where }{ }^{\prime} \text { denotes } \frac{d}{d x} .
$$

It is shown in [14] that $\operatorname{tr}(\mathcal{A})=0$ and $\operatorname{det}(\mathcal{A}) \neq 0$ and that the dislocation density tensor is given by the following symmetric matrix:

$$
S=\left(\begin{array}{ccc}
-b^{\prime}(0) & a^{\prime}(0) & 0 \\
a^{\prime}(0) & c^{\prime}(0) & 0 \\
0 & 0 & 0
\end{array}\right) .
$$

The discrete subgroup $D$ of $S_{1}$ is generated by $\boldsymbol{e}_{1}, \boldsymbol{e}_{2}, \boldsymbol{e}_{3}$. Let these vectors have matrix representations $r_{m}\left(e_{1}\right):=B, r_{m}\left(e_{2}\right):=C$ and $r_{m}\left(e_{3}\right):=A$. Note that $A$ depends on the matrix $\theta=\phi(1)$. The matrix group $D_{m}$ generated by $A, B, C$ is isomorphic to $D$. The commutators of the generating matrix elements satisfy

$$
(A, B)=B^{1-d} C^{c}, \quad(A, C)=B^{b} C^{1-a}, \quad(B, C)=0,
$$

and from this one can show that $D=\left(\mathbb{Z}^{3}, \boldsymbol{\psi}\right)$. Thus the discrete structure which is the analogue of perfect lattice $L$ is the cubic lattice $\mathbb{Z}^{3}$ with group multiplication $\boldsymbol{\psi}$ defined by (7.2).

\subsection{Symmetries of $D$}

The set of global symmetries of a discrete subgroup $D \subset S_{1}$ corresponds to the choices of three elements $\boldsymbol{g}_{1}, g_{2}, g_{3} \in D$ such that the group generated by these elements, denoted $G=g p\left(\boldsymbol{g}_{1}, \boldsymbol{g}_{2}, \boldsymbol{g}_{3}\right)$ in [14,15] equals $D$. These are the changes of generators which preserves the integer lattice $\mathbb{Z}^{3}$ in $S_{1}$. It turns out that the conditions on $g_{1}, g_{2}, g_{3}$ that are necessary and sufficient that $G=D$ are the same as those that 
are necessary and sufficient that the commutator subgroups $G^{\prime}$ and $D^{\prime}$ are equal. Here, we merely note that the elements $\left\{\boldsymbol{g}_{1}, \boldsymbol{g}_{2}, \boldsymbol{g}_{3}\right\}$ can be put into canonical form by a sequence of the following operations: permuting a pair of elements, inversion of a single element, multiplication of precisely one element by another. Then, explicit formulae given in $[14,15]$ provide conditions, on the canonical set of elements so obtained, which are necessary and sufficient in order that the set generate $D$.

\section{ELASTIC SYMMETRIES OF DEFECTIVE CRYSTALS}

In Sections 6 and 7 we have summarised results about the nature and geometrical symmetries of the discrete structures $D$ which we take to be the defective crystal analogues of the perfect lattice whose symmetries are widely used in classical crystallography. These structures $D$ are embedded in a continuous Lie group, just as a perfect lattice $L$ is embedded in $\mathbb{R}^{3}$ with addition as the group multiplication. Recall that the geometrical symmetries of a perfect lattice $L$ with basis vectors $\left\{\ell_{1}, \ell_{2}, \ell_{3}\right\}$ are the bijections of $L$ which preserve addition. These are given by $\phi_{L}\left(\ell_{a}\right)=\gamma_{a b} \ell_{b}$, $a, b=1,2,3$ where $\gamma=\left(\gamma_{a b}\right) \in \mathrm{GL}_{3}(\mathbb{Z})$. Note that these bijections $\phi_{L}: L \rightarrow L$ extend uniquely to smooth bijections of $\mathbb{R}^{3}$ that preserve addition so that every geometrical symmetry of $L$ represents a (restriction of an) elastic deformation.

In contrast, in the case of the uniform defective crystal whose discrete structures $D$ are discrete subgroups of nilpotent or solvable Lie groups, the analogous results do not hold. There is a difference between the set of 'symmetries' (the changes of generators summarised in Sections 6 and 7 which preserve the elements of $D$ ) and the subset of these symmetries which preserve the group structure of $D$ (these are the automorphisms of $D$ ). We consider now whether or not the automorphisms of $D$ extend uniquely to automorphisms of the ambient Lie group. First, an elastic deformation provides an isomorphism of Lie groups, see [18]. Recall, too, that if $\phi: G \rightarrow H$ is a Lie group homomorphism, and $\mathfrak{g}, \mathfrak{h}$ are the corresponding Lie algebras, then $\nabla \phi(\mathbf{0})$ is a Lie algebra homomorphism and

$$
\phi\left(\mathrm{e}^{(\boldsymbol{v})}\right)=\mathrm{e}^{(\nabla \boldsymbol{\phi}(\mathbf{0}) \boldsymbol{v})}, \quad \boldsymbol{v} \in \mathfrak{g},
$$

where the exponential on the left hand side is the exponential which maps $\mathfrak{g}$ to $G$ and that on the right hand side maps $\mathfrak{h}$ to $H$.

Next, the nilpotent group $G$, and $S_{1}$, are exponential groups in the sense that each group element is the exponential of some element in the corresponding algebra. So the automorphisms of $G$ and $S_{1}$ are straightforwardly calculated.

The automorphisms of discrete subgroups of $G$ and $S_{1}$ are calculated as follows. Given a discrete subgroup $D$ which is generated by the three elements $\boldsymbol{d}_{1}, \boldsymbol{d}_{2}, \boldsymbol{d}_{3}$, $i, j=1,2,3$, one may compute the subset of the geometrical symmetries which represent automorphisms of $D$ using a result of Magnus, Karrass and Solitar [11]. Thus, a mapping $\phi_{D}:\left\{\boldsymbol{d}_{1}, \boldsymbol{d}_{2}, \boldsymbol{d}_{3}\right\} \rightarrow D$ is an automorphism of $D$ if and only if for 
any word $w$ in the generators such that $w\left(\boldsymbol{d}_{1}, \boldsymbol{d}_{2}, \boldsymbol{d}_{3}\right)=\mathbf{0}$,

$w\left(\phi_{D}\left(\boldsymbol{d}_{1}\right), \boldsymbol{\phi}_{D}\left(\boldsymbol{d}_{2}\right), \boldsymbol{\phi}_{D}\left(\boldsymbol{d}_{3}\right)\right)=0$ and $w\left(\boldsymbol{\phi}_{D}^{-1}\left(\boldsymbol{d}_{1}\right), \boldsymbol{\phi}_{D}^{-1}\left(\boldsymbol{d}_{2}\right), \boldsymbol{\phi}_{D}^{-1}\left(\boldsymbol{d}_{3}\right)\right)=0$,

where $\phi_{D}^{-1}$ is the mapping that takes $\phi_{D}\left(d_{1}\right)$ to $d_{1}, \phi_{D}\left(d_{2}\right)$ to $d_{2}$ and $\phi_{D}\left(d_{3}\right)$ to $d_{3}$. Thus, we determine the conditions that must be satisfied by $\phi_{D}$ by taking the words $w$ in (8.2) to be the relevant commutator relations.

\subsection{Automorphisms of nilpotent discrete subgroups}

Let $D \subset J$ be the discrete subgroup generated by the canonical set of elements $c_{1}, c_{2}, c_{3}$ with $\left(c_{1}, c_{2}\right)=c_{3}^{k}$ and $\left(c_{1}, c_{3}\right)=\left(c_{2}, c_{3}\right)=\mathbf{0}$. From (8.1) the automorphisms of $D$ (and $J$ ) are linear mappings since $\mathrm{e}^{(\boldsymbol{x})}=\boldsymbol{x}$ in $J$. It is shown in [18] that the automorphisms of $D$ have the form $\phi_{D}: D \rightarrow D$ where $\phi\left(c_{i}\right)=c_{1}^{\alpha_{i}} c_{2}^{\beta_{i}} c_{3}^{\gamma_{i}}$, $i=1,2,3$ and

$$
\left(\begin{array}{lll}
\alpha_{1} & \alpha_{2} & \alpha_{3} \\
\beta_{1} & \beta_{2} & \beta_{3} \\
\gamma_{1} & \gamma_{2} & \gamma_{3}
\end{array}\right)=\left(\begin{array}{lll}
\alpha_{1} & \alpha_{2} & 0 \\
\beta_{1} & \beta_{2} & 0 \\
\gamma_{1} & \gamma_{2} & \epsilon
\end{array}\right)
$$

where $\epsilon= \pm 1, \alpha_{1} \beta_{2}-\alpha_{2} \beta_{1}=\epsilon$ and $\gamma_{1}, \gamma_{2}$ are arbitrary integers.

In this case, a theorem of Mal'cev [12] states that every automorphism of $D$ extends uniquely to an automorphism of $J$. Thus every symmetry of $D$ which corresponds to an automorphism of $D$ is an elastic symmetry of $D$ in that it is the restriction of an elastic deformation of $J$. Symmetries of $D$ which do not satisfy (8.2) cannot be extended to automorphisms of $J$, so they may be called inelastic symmetries.

\subsection{Automorphisms of solvable discrete subgroups}

In the recent work [13] we showed that $\phi_{D}: D_{m} \rightarrow D_{m}$ is an automorphism if $\phi_{D}(A)=A^{\alpha_{1}} B^{\beta_{1}} C^{\lambda_{1}}, \phi_{D}(B)=A^{\alpha_{2}} B^{\beta_{2}} C^{\lambda_{2}}, \phi_{D}(C)=A^{\alpha_{3}} B^{\beta_{3}} C^{\lambda_{3}}$, where $\alpha_{1}=\xi= \pm 1, \alpha_{2}=\alpha_{3}=0, \beta_{1}, \gamma_{1}$ arbitrary and

$$
\chi:=\left(\begin{array}{ll}
\beta_{2} & \beta_{3} \\
\gamma_{2} & \gamma_{3}
\end{array}\right) \in \mathrm{GL}_{2}(\mathbb{Z}) \text { is such that } \theta^{\xi} \chi=\chi \theta .
$$

There is an algorithm for determining the matrices $\chi$ which satisfy (8.3) for a given matrix $\theta$, described in [13], following the method used in [2]. Note that from (7.2) and (7.5), it is the dislocation density tensor that determines the automorphisms of $D$, via (8.3). Aut $(D)$ is a finitely generated infinite group.

In the case where $D$ is a uniform discrete subgroup of $S_{1}$ a theorem of Gorbatsevich [10] implies that every automorphism of $D$ extends uniquely to an automorphism of $S_{1}$. The automorphism of the discrete group $D$ are therefore restrictions of elastic deformations. 


\section{ACKNOWLEDGEMENT}

We acknowledge the support of the UK Engineering and Physical Sciences Research Council through grant EP/G047162/1.

\section{REFERENCES}

[1] L. Auslander, L. Green, and F. Hahn, Flows on homogeneous spaces, ser. Ann. Math. Stud. Princeton, NJ: Princeton University Press, 1963, vol. 53.

[2] M. Baake and J. A. G. Roberts, "Reversing symmetry group of $G L(2, \mathbb{Z})$ and $P G L(2, \mathbb{Z})$ matrices with connections to cat maps and trace maps," J. Phys. A: Math. Gen., vol. 30, pp. 1549-73, 1997.

[3] P. Cermelli and G. P. Parry, "The structure of uniform discrete defective crystals," Continuum Mechanics and Thermodynamics, vol. 18, pp. 47-61, 2006.

[4] M. Chipot and D. Kinderlehrer, "Equilibrium configurations of crystals," Arch. Rat. Mech. Anal., vol. 103 , pp. 237-277, 1988.

[5] C. Davini, "A proposal for a continuum theory of defective crystals," Arch. Rat. Mech. Anal., vol. 96, pp. 295-317, 1986.

[6] C. Davini and G. P. Parry, "On defect preserving deformations in crystals," Int. J. Plasticity, vol. 5, pp. 337-369, 1989.

[7] C. Davini and G. P. Parry, "A complete list of invariants for defective crystals," Proc. Roy. Soc. London A, vol. 432, pp. 341-365, 1991.

[8] M. Elzanowski and G. P. Parry, "Material symmetry in a theory of continuously defective crystals," J. Elasticity, vol. 74, pp. 215-237, 2004.

[9] I. Fonseca and G. P. Parry, "Equilibrium configuration of defective crystals," Arch. Rat. Mech. Anal., vol. 120, pp. 245-283, 1992.

[10] V. V. Gorbatsevich, "Lattices in solvable lie groups and deformations of homogeneous spaces," Math. USSR - Sb, vol. 20, pp. 249-266, 1973.

[11] W. Magnus, A. Karrass, and D. Solitar, Combinatorial Group Theory. New York: Dover, 1976.

[12] A. Mal'cev, "On a class of homogeneous spaces," Izv. Akad. Nauk SSSR, Ser. Mat., vol. 13, pp. 9-32, 1949, am. Math. Soc. Translation, 39, (1949).

[13] R. Nicks and G. P. Parry, "Group elastic symmetries common to continuum and discrete defective crystals," in preparation.

[14] R. Nicks and G. P. Parry, "On symmetries of crystals with defects related to a class of solvable groups $\left(S_{1}\right)$, , Math. Mech. Solids, vol. 17, pp. 631-651, 2011.

[15] R. Nicks and G. P. Parry, "On symmetries of crystals with defects related to a class of solvable groups $\left(S_{2}\right)$, , Mathematical Methods in the Applied Sciences, vol. 35, pp. 1741-1755, 2012.

[16] P. J. Olver, Equivalence, invariants, and symmetry. Cambridge: Cambridge University Press, 1996.

[17] G. P. Parry, "Group properties of defective crystal structures," Mathematics and Mechanics of Solids, vol. 8, pp. 515-538, 2003.

[18] G. P. Parry, "Elastic Symmetries of Defective Crystals,” J. Elasticity, vol. 101, pp. 101-120, 2010.

[19] G. P. Parry and R. Sigrist, "Reconciliation of local and global symmetries for a class of crystals with defects," J. Elasticity, vol. 107, pp. 81-104, 2012.

[20] M. Pitteri and G. Zanzotto, Continuum Models for Phase Transitions and Twinning in Crystals. Boca Raton, London, New York, Washington DC: Chapman and Hall, 2003.

[21] L. S. Pontryagin, Topological Groups, 2nd ed. New York: Gordon and breach, 1955.

[22] W. Thurston, Three Dimensional Geometry and Topology, vol 1. Princeton: Princeton Univ. Press, 1997. 
Authors' addresses

\section{Rachel Nicks}

School of Mathematical Sciences, University of Nottingham, Nottingham, NG7 2RD, UK

E-mail address: rachel.nicks@nottingham.ac.uk

Gareth Parry

School of Mathematical Sciences, University of Nottingham, Nottingham, NG7 2RD, UK

E-mail address: gareth.parryenottingham.ac.uk 